\title{
Article \\ Experimental Study on Support Vector Machine-Based Early Detection for Sensor Faults and Operator-Based Robust Fault Tolerant Control
}

\author{
Mingcong Deng* $*$, Yuki Tanaka and Ximei Li
}

check for

updates

Citation: Deng, M.; Tanaka, Y.; Li, X.

Experimental Study on Support

Vector Machine-Based Early

Detection for Sensor Faults and

Operator-Based Robust Fault

Tolerant Control. Machines 2022, 10,

123. https://doi.org/10.3390/

machines10020123

Academic Editor: Christoph

M. Hackl

Received: 20 December 2021

Accepted: 7 February 2022

Published: 10 February 2022

Publisher's Note: MDPI stays neutral with regard to jurisdictional claims in published maps and institutional affiliations.

Copyright: (C) 2022 by the authors. Licensee MDPI, Basel, Switzerland. This article is an open access article distributed under the terms and conditions of the Creative Commons Attribution (CC BY) license (https:/ / creativecommons.org/licenses/by/ $4.0 /)$.

\author{
Department of Electrical and Electronic Engineering, The Graduate School of Engineering, Tokyo University of \\ Agriculture and Technology, Tokyo 184-8588, Japan; s150035y@st.go.tuat.ac.jp (Y.T.); \\ s341484s@st.go.tuat.ac.jp (X.L.) \\ * Correspondence: deng@cc.tuat.ac.jp; Tel.: +81-42-388-7134
}

\begin{abstract}
Considering sensor faults for a thermoelectric cooler actuated by Peltier devices, this work proposes an operator-based robust nonlinear fault tolerant controller (FTC) integrated with early fault detection using a support vector machine (SVM). Firstly, a physical model is formulated based on the law of heat transfer, and the estimated model is derived based on Volterra identification. Then, an operator-based robust nonlinear control system is employed to compensate for uncertainties and to eliminate the effects of coupling. Furthermore, FTC integrated with SVM-based early fault detection is designed to improve the safety performance in the case of sensor faults. The simulation results indicate that SVM-based fault detection can shorten the detection time in comparison to the conventional method without the SVM classier. The experiment results are utilized to verify the tracking performance of the proposed FTC method in the case study.
\end{abstract}

Keywords: operator theory; robust nonlinear control; fault tolerant control; support vector machine; fault detection; Peltier cooler

\section{Introduction}

Thermoelectric technology has been growing rapidly with increasing significance, and it requires precise temperature regulation for the physical, chemical, and biotechnological reaction, using the relevant heating/cooling technique. The thermoelectric cooler (TEC) actuated by Peltier devices has a high-surface-to-volume area with an efficient transfer and higher safety level, and it is regarded as a promising new tool for studying and optimizing fine medicine chemicals. Peltier as a solid-state construction is usually applied in the thermoelectric cooler, which transfers heat from one side to the other, depending on the direction of the current. Peltier devices have few noticeable advantages: no moving parts, no effects of noises and vibrations, able to operate without Freon gas, light weighted with a long-life expectancy, etc. It is also worth noting that the thermoelectric cooler is a classical nonlinear dynamic system that has parameter uncertainties and comes with the effects of coupling between adjacent devices. As TEC has the nature of parameter uncertainties, process disturbance, and measured noises, it is difficult to obtain the exact mathematical-mechanical modelling. However, nothing comes without consequences. Usually, automated systems are more likely to be more vulnerable to cope with potential faults, if there are any. The faults placed in a certain area could lead to massive consequences, and lower the economic efficiency and would reduce the product availability and reliability. The safety-critical systems such as aircraft flight control, spacecraft, robots, power plants, and thermal processes are extremely vulnerable to faults, and the requirement for stability/reliability means no tolerance to the fault. To solve the above issues, basic problems are concerned with modelling; robust stability and estimation; output performance; and safety in dynamic linear/nonlinear systems associated with uncertainty, time-delay, hysteresis, perturbation, etc. [1-4]. 
In previous research, the microreactor actuated by Peltier devices aimed to achieve a level of guaranteed stability, while minimizing the influence of disturbances, noises, and perturbations, and optimizing the safety performance. In the initial stage, the primary focus is to achieve robust stability for the tracking performance. In this paper, the research focuses on operator-based design methods. However, in this approach, some open problems using the existing results, such as robust filtering, dynamic environment analysis, adaptive control, and predictive control, need to be considered [5,6]. An operator-based roust right coprime factorization for nonlinear feedback control systems has been a promising approach for nonlinear systems analysis, design, stabilization, and control. Moreover, the robust stability of the nonlinear systems can be guaranteed by the establishment of the Bezout identity and an inequality in the form of the Lipschitz norm [7-13]. An operatorbased robust controller for nonlinear systems with the Prandtl-Ishlinskii hysteresis is better than the Lyapunov method, for which simulation results have confirmed that the vibration of the flexible arm converges much faster than the system compared with the Lyapunov method [14]. The robust right coprime factorization theory can be utilized to detect the fault signal in the tracking operator system. The Bezout identity was used to ensure that the observed fault signal equals the real fault signal in the literature [15,16]. Kawahata et al. [17] extended the MIMO microreactor considering the compensation of uncertainties and the elimination of interference, achieving nonlinear cooling control based on operator theory and fault tolerant control when switching the close feedback loop. When a fault occurs, the compensation operator for the fault signal will be active. Furthermore, to prevent dangerous accidents when optimizing the performance safety level, many different control strategies are considered during the process of corresponding to different fault levels. While FTC is a real-time safety-critical system that operates well within its' feasible limits, fault detection is an indicator that makes a binary decision regarding whether the fault occurs or not by checking the residual signal (i.e., the difference between the real system and the normal model behaviors). The integrated fault detection and fault-tolerant control for a Hexacopter is proposed to handle up to two actuator failures in the presence of disturbances [18]. Salahshoor et al. [19] utilized the fusion of an SVM classifier with an ANFIS (adaptive neuro-fuzzy inference system) classifier, integrated into a common framework to enhance the fault detection and diagnostic tasks. Furukawa et al. [20] presented a fault detection method when using SVM combined with ChangeFinder for a tank-system. As mentioned above, it is worth noting that, although there is much existing literature discussing the fault tolerant control and fault detection matter separately, current research has made few attempts towards a fault tolerant controller dealing with the delay detection in nonlinear dynamic system, and no existing literature so far has specifically discussed the combination of operator-based robust nonlinear FTC with early fault detection.

The main contribution of this paper is to propose a fault tolerant controller integrated with SVM-based early detection for multiple sensor faults. The technical novelty focuses on a robust nonlinear FTC method that reduces the fault detection time under different faulty conditions. That is, SVM as a two-kind classifier has been employed to deal with noised uncertain nonlinear processes. As a result, earlier detection is obtained than from the former methods, where SVM-based fault detection improves the sensitivity classification against the noise and uncertainties and shortens the detection time. If the detecting scheme finds a fault, the fault tolerant control is switched by an operator, and the employed control is based on Volterra identification, where an operator-based robust nonlinear control system is designed to compensate for uncertainties and to eliminate the coupling effect. The remainder of this paper is organized as follows. Section 2 will illustrate the preliminaries and problem statement. The structure of the microreactor system is described as the object research, the physical model is formulated based on the laws of heat transfer and the estimated model is derived by the Volterra identification in Section 3. The proposed framework of the robust nonlinear control system design is carried out in Section 4. The simulation and experiment results are presented in Section 5. Section 6 draws the conclusions. 


\section{Mathematical Preliminaries}

Some necessary notations and definitions about operator theory, Volterra identification, and support vector machines are formulated as the fundamentals of the control system in this section.

\subsection{Operator Theory}

The definition of nonlinear operators is given [21] by R. de Figueiredo and G. Chen.

Let $U$ and $Y$ be linear spaces over the field of complex numbers. Let $P: U \rightarrow Y$ be an operator mapping from $U$ to $Y$, and denote the domain and range of $P$ by $D(P) \subseteq U$ and $R(P) \subseteq Y$, respectively.

The concept of right coprime factorization is as follows.

Let $P: D(P) \rightarrow R(P)$ be a causal and stabilizabling nonlinear operator. $P$ is said to have a right coprime factorization on $D(P)$ over the space of finite-gain stable and causal operators if it has a right factorization $P=N D^{-1}$ on $D(P)$. Moreover, two operators exist, $A: R(N) \rightarrow D(P), B: R(D) \rightarrow D(P)$, and for the unimodular operator $M: D(N) \rightarrow D(P)$, we have the Bezout identity:

$$
A N+B D=M, M \in(W, U)
$$

\subsection{Volterra Identification}

Volterra identification aims at developing high-fidelity mathematical models in the presence of nonlinearity and uncertainty from input and output measurements performed on the real structure. In mathematics, the Volterra model can be expressed as a symbolically nonlinear dynamic time-invariant functional expansion by an infinite series of multidimensional convolution integrals. The Volterra series constructs an explicit input-output relationship for nonlinear dynamic systems.

For a nonlinear continuous time-invariant system with input $u(t)$ and the system response $y(t)$, the Volterra model can be expanded symbolically by a series of convolution integrals,

$$
\begin{gathered}
\mathrm{y}(t)=h_{0}+\int_{-\infty}^{\infty} h_{1}(\tau) u(t-\tau) d \tau+\int_{-\infty}^{\infty} \int_{-\infty}^{\infty} h_{2}\left(\tau_{1}, \tau_{2}\right) \Pi_{i=1}^{2} u\left(t-\tau_{i}\right) d \tau_{i}+\ldots+ \\
\int_{-\infty}^{\infty} \int_{-\infty}^{\infty} \cdots \int_{-\infty}^{\infty} h_{n}\left(\tau_{1}, \tau_{2}, \cdots, \tau_{n}\right) \Pi_{i=1}^{n} u\left(t-\tau_{i}\right) d \tau_{i}+\cdots
\end{gathered}
$$

which is also known as the Volterra series. In Equation (2), each of the convolution integrals contains a kernel, either linear $\left(h_{1}\right)$ or nonlinear $\left(h_{2} \ldots h_{n}\right)$, which represent the behavior of the system. Generally, the first-order kernel $h_{1}(\tau)$ is the linear response associated with input signal $u$ at time lag $t$, and $h_{2}\left(\tau_{1}, \tau_{2}\right)$ is the second-order kernel that reflects the quadratic properties. The rest may be deduced by analogy, $h_{n}\left(\tau_{1}, \tau_{2}, \ldots \tau_{n}\right)$ is the $n$th order kernel and contains the $n$th order nonlinear information of the system.

\subsection{Support Vector Machine}

SVM is a set of supervised learning methods used for classification, regression, and outlier detection [22,23]. Real-time fault detection using the generalized Gaussian function as the kernel function of SVM is presented in [24]. In dealing with the noise-containing nonlinear process, the SVM classifier has been widely employed for sensitivity classification against noise in fault detection.

The classifier $g(x)$ is defined as follows, using the real-valued decision function $f(x)$.

$$
g(x)=\left\{\begin{aligned}
1, & f(x)>1 \\
-1, f(x) & <1
\end{aligned}\right.
$$

here, $f(x)$ is a linear function, and the division of hyperplanes can be described by linear equations:

$$
f(x)=w^{T} x+b
$$

where $w=\left(w_{1} ; w_{2} ; \ldots ; w_{d}\right)$ is weight vector, $x$ is the input vector, and $b$ is the basis. 


\section{Modeling of Microreactor System}

As shown in Figure 1, the microreactor system can generally be divided into three parts: actuators (i.e., Peltier device), the plant (i.e., the tube of water), and sensors (i.e., the tube sensors are embedded the inner aluminum boxes and the aluminum sensors are installed the outer aluminum plates). Peltier devices with size $3 \times 3 \mathrm{~cm}^{2}$ are used to cool water in the inner tube. The operating temperature ranges from $-55^{\circ} \mathrm{C}$ to $+80^{\circ} \mathrm{C}$, with accuracy and good long-term stability. A large surface-to-volume ratio also allows for better thermal control. Four Peltier devices are pasted on the front and back sides of each of the aluminum boxes. The parameters of the thermal transfer and microreactor model are described in Tables 1 and 2, respectively. $S_{4}$ indicates the Peltier device's surface area and the tube's radius is $r_{1}=0.01 \mathrm{~m}$.

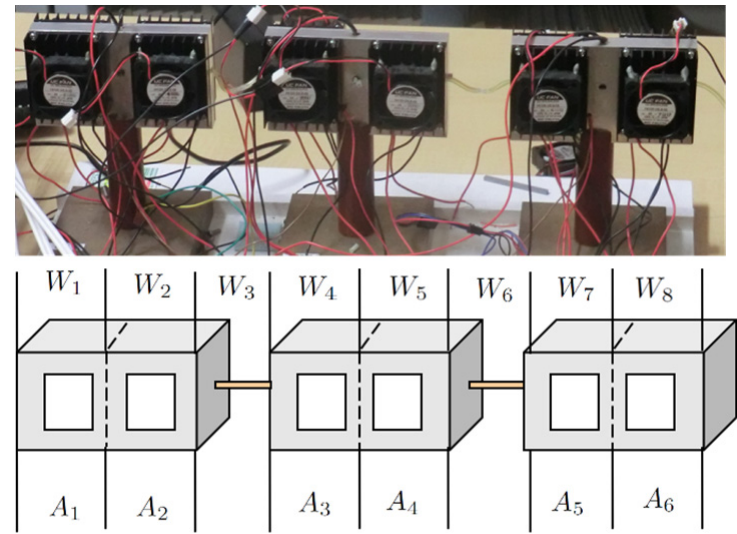

$\begin{array}{lll}\text { Box } 1 & \text { Box } 2 & \text { Box } 3\end{array}$

Figure 1. Photos and configuration of the microreactor system.

Table 1. Parameters of the microreactor system.

\begin{tabular}{cccc}
\hline Parameters & Value[m] & Parameters & Value[m] \\
\hline$d_{1}$ & 0.07 & $d_{5}$ & 0.045 \\
$d_{2}$ & 0.12 & $d_{6}$ & 0.02 \\
$d_{3}$ & 0.014 & $d_{7}$ & 0.01 \\
$d_{4}$ & 0.03 & $d_{8}$ & 0.06 \\
\hline
\end{tabular}

Table 2. Parameters of the thermal transfer.

\begin{tabular}{ccc}
\hline Symbol & Description & Unit \\
\hline$T_{0}$ & Initial temperature & {$[\mathrm{K}]$} \\
$T_{a_{n}}$ & Aluminum temperature of Part $A_{n}$ & {$[\mathrm{~K}]$} \\
$T_{w_{n}}$ & Water temperature of Part $W_{n}$ & {$[\mathrm{~K}]$} \\
$S$ & Seebeck coefficient & {$[\mathrm{V} / \mathrm{K}]$} \\
$\alpha$ & Thermal transfer rate & {$\left[\mathrm{W} / \mathrm{m}^{2} \cdot \mathrm{K}\right]$} \\
$\lambda$ & Thermal conductivity rate & {$\left[\mathrm{W} / \mathrm{m}^{2} \cdot \mathrm{K}\right]$} \\
$c$ & Specific heat capacity & {$[\mathrm{J} / \mathrm{kg} \cdot \mathrm{K}]$} \\
$\epsilon$ & Emissivity & - \\
$\sigma$ & Stefan-Boltzmann constant & {$\left[\mathrm{W} / \mathrm{m}^{2} \cdot \mathrm{K}^{4}\right]$} \\
$I$ & Current & {$[\mathrm{A}]$} \\
$R$ & Peltier's resistance & {$[\Omega]$} \\
$m$ & mass & {$[\mathrm{kg}]$} \\
\hline
\end{tabular}

\subsection{Physical Model Based on Laws of Heat Transfer}

Models of the single aluminum plate and tube are presented in Figure 2, based on the physical laws of heat transfer, such as Newton's law of cooling, Fourier's law concerning 
thermal conduction, and Joule heat. As mentioned above, Newton's Law of cooling states that the rate of change of temperature is proportional to the difference between the temperature of the object and that of the surrounding environment. Fourier's law is known as the rate of heat conduction through a plane layer, and is proportional to the temperature gradient across the layer and the heat transfer area of the layer. Joule heating is the physical effect through which the current passing through an electrical conductor produces thermal energy. The thermoelectric effect is the actuated thermal process by Peltier devices, as shown in Figure 3.

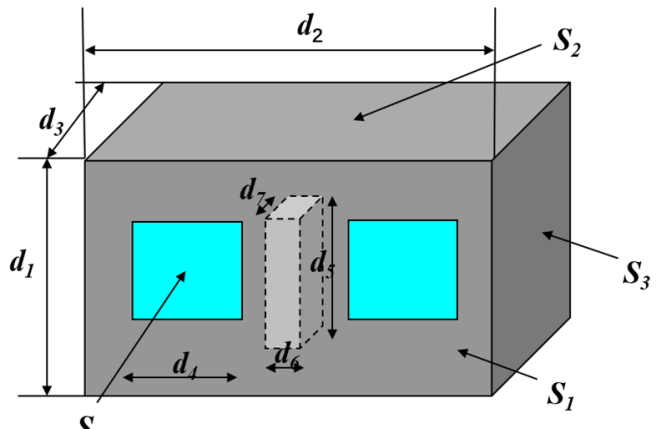

$\boldsymbol{S}_{4}$

(1) Model of aluminum plate

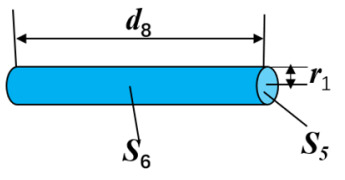

(2) Model of tube

Figure 2. Model of a single aluminum plate and tube.

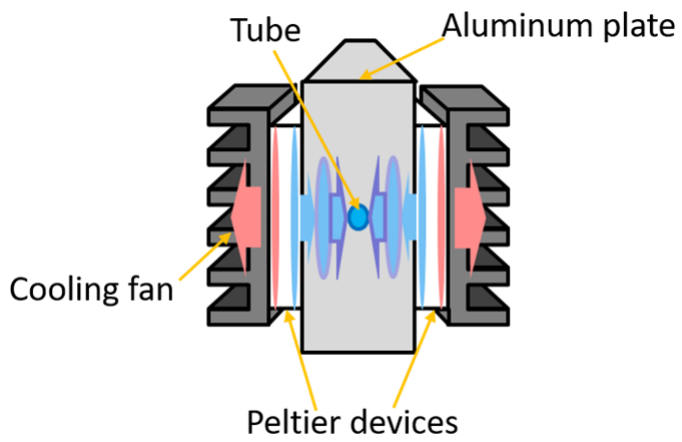

Figure 3. Peltier devices actuated the thermal transfer process.

\subsubsection{Modeling of Peltier Device}

Quantity of endothermic of Peltier devices $u_{d_{1}}$ is formulated by Equation (3):

$$
u_{d_{1}}=S T_{c} I-\lambda \Delta T A-\frac{1}{2} R I^{2}
$$

where $S T_{c} I$ is derived from the Seebeck effect, $\lambda \Delta T A$ is denoted as the Newton's cooling law, and $R I^{2}$ is the Joule heat.

\subsubsection{Modeling of Aluminum Plate}

The quantity of the endothermic of the aluminum plate in Part $A_{1}$ is formulated by Equation (4). Each part on the right side of the equal sign is denoted by the endothermic of the Peltier, heat transfer of the water-aluminum plate, thermal conduction of the aluminum plate, thermal conduction of air, and thermal radiation of the aluminum plate, respectively.

$$
\begin{aligned}
& \frac{d\left(T_{0}-T_{a_{1}}\right) m_{a} c_{a}}{d t}=2 u_{d 1}+\alpha_{w} S_{6}\left(T_{a_{1}}-T_{w_{1}}\right) \\
& +\frac{\lambda_{\alpha} S_{3}\left(T_{a_{1}}-T_{a_{2}}\right)}{d x}-\alpha\left(T_{0}-T_{a_{1}}\right)\left(S_{1}+S_{2}+S_{3}-2 S_{4}-S_{5}\right) \\
& +\epsilon_{a} \sigma\left(T_{a_{1}}^{4}-T_{0}^{4}\right)\left(S_{1}+S_{2}+S_{3}-2 S_{4}-S_{5}\right)
\end{aligned}
$$


Assuming that

$$
y_{a_{n}}=T_{0}-T_{a_{n}}
$$

The quantity of endothermic of the aluminum plate of Part $A_{n}$ can be transfer as Equation (6).

$$
\frac{d y_{a_{n}}}{d t}=\omega_{a_{n}}+\sum_{m=1}^{4}(-1)^{m} A_{a_{n m}} y_{a_{n}}^{m} n=1 \sim 6
$$

\subsubsection{Modeling of Tube}

The quantity of endothermic of the tube of Part $W_{1}$ is formulated by Equation (7) based on the heat transfer of water and the thermal conduction of tube $W_{1}$.

$$
\begin{aligned}
& \frac{d\left(T_{0}-T_{w_{1}}\right) m_{w} c_{w}}{d t}=\alpha_{w} S_{6}\left(T_{w_{1}}-T_{a_{1}}\right) \\
& -\frac{\lambda_{w} S_{5}\left\{\left(T_{w_{0}}-T_{w_{1}}\right)-\left(T_{w_{0}}-T_{w_{2}}\right)\right\}}{d x}
\end{aligned}
$$

The quantity of endothermic of the tube of Part $W_{3}$ is formulated by Equation (8) based on the thermal radiation and thermal conduction of the tube $W_{3}$.

$$
\begin{aligned}
& \frac{d\left(T_{0}-T_{w_{3}}\right) m_{w} c_{w}}{d t}=\epsilon_{w} \sigma\left(T_{w_{3}}^{4}-T_{0}^{4}\right) S_{6} \\
& -\frac{\lambda_{w} S_{5}\left\{2\left(T_{w_{0}}-T_{w_{3}}\right)-\left(T_{w_{0}}-T_{w_{2}}\right)-\left(T_{w_{0}}-T_{w_{4}}\right)\right\}}{d x}
\end{aligned}
$$

Assuming that

$$
y_{w_{k}}=T_{0}-T_{w_{k}}
$$

The quantity of endothermic of the tube of Part $W_{n}$ can be transferred as follows.

$$
\begin{aligned}
& \frac{d y_{w_{k}}}{d t}=\omega_{w_{k}}-A_{w_{k}} y_{w_{k}} k=1,2,4,5,7,8 \\
& \frac{d y_{w_{k}}}{d t}=\omega_{w_{k}}+\sum_{m=1}^{4}(-1)^{m} A_{w_{k m}} y_{w_{k}}^{m} k=3,6
\end{aligned}
$$

\subsection{The Estimated Model by Volterra Identification}

The Volterra kernels are the backbone of the Volterra series. All of the system characteristic related information is contained in these kernels. For any system with its Volterra kernels, the Volterra series can predict the system's response to any arbitrary input. For any system with its Volterra kernels, the Volterra series can predict the system's response to any arbitrary input. One issue is obtaining Volterra kernels using M-sequences and the correlation technique. Due to the weak nonlinearity, the estimated model of the microreactor system can be represented by one order Volterra series. The computation of the Volterra identification is testified by the simulation results in Figures 4 and 5 . The cross-correlation function of an input signal and output signal is transformed to a kernel while the autocorrelation function $\varphi_{u u}$ is equal to the impulse signal $\delta$ when the maximum length sequence signal (M-seq) is the input.

$$
\begin{gathered}
\varphi_{u y}(\tau)=\int_{0}^{\infty} g(\tau) \varphi_{u u}(t-\tau) d \tau \\
\varphi_{u y}(t)=g(t)
\end{gathered}
$$



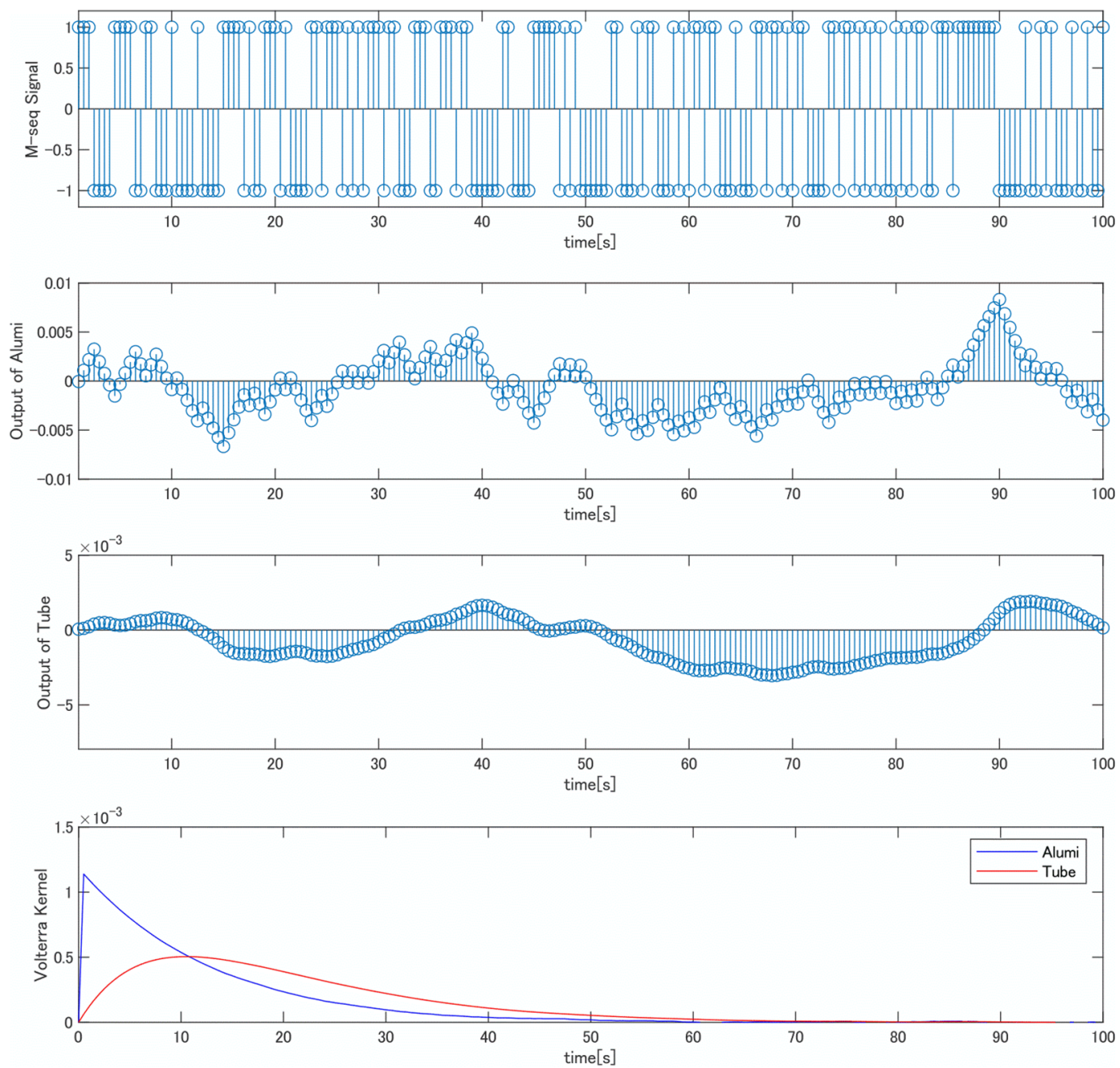

Figure 4. Identification of Volterra kernels.
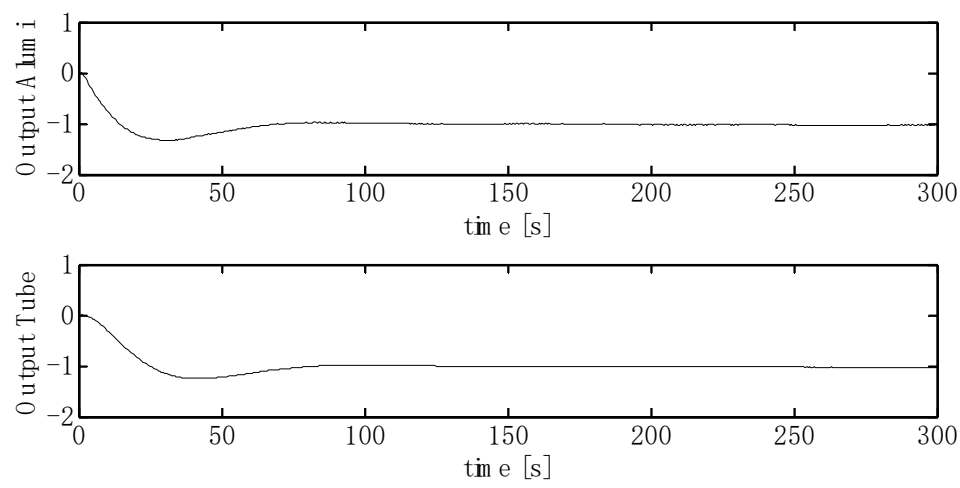

Figure 5. The temperature outputs of the aluminum plate and tube.

According to Equations (10) and (11), the kernel identification of the aluminum plate and tube are computed by the sensor measurement as the output and M-seq as the input signal, respectively, as shown in Figure 4.

Then, the output of aluminum plate and tube are estimated based on Equation (12), where the input $u(t)$ is the current signal and the output $y(t)$ is the sensor measurement. The results are presented in Figure 5.

$$
y(t)=\int_{0}^{\infty} g(\tau) u(t-\tau) d \tau
$$




\section{Control System Design}

In this section, the proposed control system is designed as five blocks; while the three blocks of operator-based robust nonlinear control, early fault detection, and fault tolerant control are introduced in detail, for the remaining blocks of compensation of uncertainties and elimination of coupling effects, please refer to Deng, M. and Koyama, A. [25].

\subsection{Operator-Based Robust Nonlinear Control}

Figure 6 shows the operator-based temperature tracking control system.

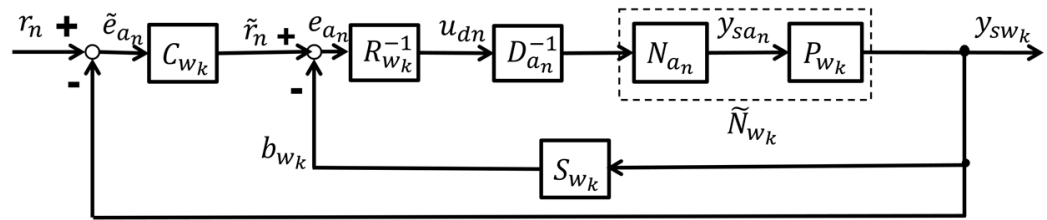

Figure 6. Operator-based temperature tracking control system.

Operators $D_{a_{n}}$ and $\widetilde{N}_{W_{k}}$ are rewritten as the given plant by right coprime factorization. Operators $R_{W_{k}}$ and $S_{W_{k}}$ are designed by using the arbitrary constant to satisfy the Bezout identify in Equation (13). Then, the cooling temperature control system is said to be BIBO stable.

$$
S_{W_{k}} \widetilde{N}_{W_{k}}+R_{W_{k}} D_{a_{n}}=I,(I \text { : identity mapping })
$$

the operators $S_{W_{k}}$ and $R_{W_{k}}$ are obtained as below.

$$
\begin{gathered}
S_{W_{k}}\left(y_{s w_{k}}\right)=\left(1-B_{n}\right)\left(\widetilde{N}_{W_{k}}^{-1}\left(y_{s a_{n}}\right)\right) \\
R_{W_{k}}\left(u_{d_{n}}\right)=\frac{B_{n}}{m_{a} c_{a}} u_{d_{n}}
\end{gathered}
$$

The tracking controller $C_{w_{k}}$ is designed as follows:

$$
C_{w_{k}}\left(\widetilde{e}_{a_{n}}\right)=K_{P_{n}} \widetilde{e}_{a_{n}}+K_{I_{n}} \int_{0}^{t} \widetilde{e}_{a_{n}}(\tau) d \tau
$$

\subsection{SVM-Based Early Fault Detection}

Early fault detection is based on the SVM algorithm in order to make the process safe, more efficient, and more economical if abnormal behavior is noticed in a timely manner. The fundamental principle of the SVM algorithm is separating the dataset into two classes (normal or faulty condition) according to the hyperplane (a decision boundary), which should have maximum distance between support vectors (i.e., representative training data point)) in each class. The basic process of constructing an SVM classifier model is as follows: using the optimal parameters and appropriate RBF kernel to build a classifier based on the training set, then using it to predict the target value of the data in testing set. Operator $Q$ of the fault tolerant control system works in the occurrence of faults and outputs a compensation signal $q$ when it detects a fault in the temperature sensor (tube).

From the viewpoint of nonlinear classification, the radial basis function kernel (RBF) is determined using Equation (14).

$$
K\left(x_{i}, x_{j}\right)=\exp \left(-\gamma\left\|x_{i}-x_{j}\right\|^{2}\right)
$$

The hyper-parameters of the RBF kernel $\mathrm{C}$ and $\gamma$ are obtained by simulation and real experimental tests, respectively. The heat absorption amount is learned as the input, and the tube temperature sensor value $y_{\text {swk }}$ is learned as the output. Therefore, the time series data are also trained for the output. The SVM software uses libsvm, which is common for the simulation and the real machine experiment. Figure 7 shows early detection using SVM 
and operator $Q$ behind the tracking controller design, uncertainties compensation, and coupling effects elimination.

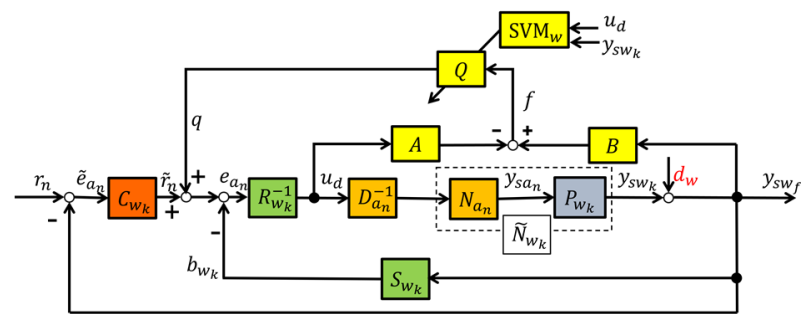

Figure 7. Early fault detection using SVM.

Assume a fault $d_{w}$ in the temperature sensor of the tube. Operator $Q$ is designed to satisfy the Bezout identity for eliminating the effect of fault $d_{w}$. First, the control loop of the fault condition is formulated in Equation (15).

$$
R_{w_{k}} D_{a_{n}}(\widetilde{\omega})=C_{w_{k}}\left(e_{a_{n}}\right)+Q(f)-S_{w_{k}}\left(y_{s w f}\right)
$$

The fault signal $f$ in Equation (16) is calculated from the actual temperature sensor value and the input.

$$
f(t)=B \widetilde{N}_{w_{k}}-A D_{a_{n}}
$$

Formula (15) is converted to the following equation:

$$
\left(S_{w_{k}} \widetilde{N}_{w_{k}}-Q B \widetilde{N}_{w_{k}}\right) \widetilde{N}_{w_{k}}^{-1}\left(y_{s w f}\right)=C_{w_{k}}\left(e_{a_{n}}\right)-\left(R_{w_{k}} D_{a_{n}}+Q A D_{a_{n}}\right) \widetilde{N}_{w_{k}}^{-1}\left(y_{s w f}-d_{w}\right)
$$

where the Bezout identify I and the fault $d_{w}$ are satisfied through the following conditions,

$$
\left\{\begin{array}{l}
S_{w_{k}} \widetilde{N}_{w_{k}}-Q B \widetilde{N}_{w_{k}}=I \\
R_{w_{k}} D_{a_{n}}+Q A D_{a_{n}}=0
\end{array}\right.
$$

Operators $A, B$, and $Q$ are obtained according to the below equations, where $M$ is the tracking controller:

$$
\begin{gathered}
A=D_{a_{n}}^{-1} N_{a_{n}}, B^{-1}=P_{w_{k}} \\
Q=-M R_{w_{k}} D_{a_{n}} P_{w_{k}}(f)
\end{gathered}
$$

\subsection{Fault Tolerant Control System}

As shown in Figure 8, the fault tolerant control design is derived by the differences of the tube's temperature values among the physical model based on the law of heat transfer $\left(y_{0}\right)$, the estimated value $\left(y_{1}\right)$ from the aluminum sensor and the estimated value $\left(y_{2}\right)$ from the tube sensor, and the estimated value of Volterra identification $\left(y_{3}\right)$. The equation of $W a$ is used to detect and remove the faulty sensor using the weight of the estimated values, which are denoted as below. The output of the fault tolerant control $W_{a}$ can be calculated based on Equation (18), where there is a condition $y_{0} \neq y_{\mathrm{i}}$ at all times.

$$
W_{a}=\frac{1}{\sum_{i=1}^{3} \frac{1}{\left(y_{0}-y_{i}\right)^{2}}} \sum_{i=1}^{3} \frac{1}{\left(y_{0}-y_{i}\right)^{2}} y_{i}
$$




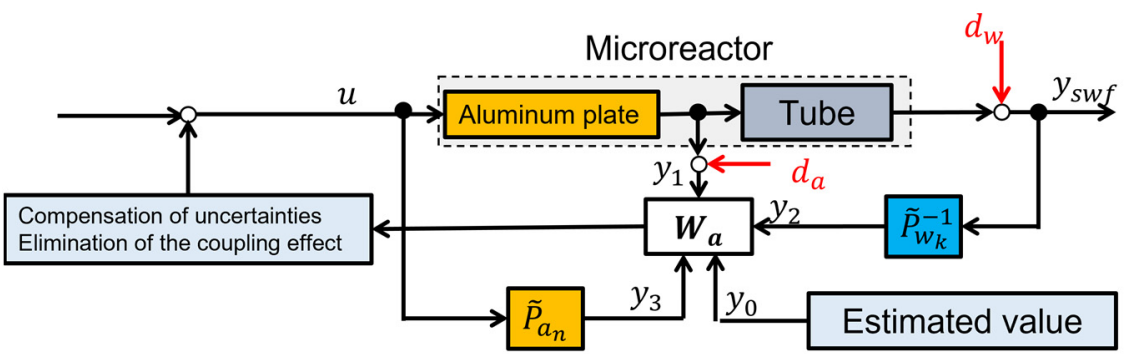

Figure 8. Illustration of fault tolerant control.

Operator-based robust nonlinear control is achieved the temperature tracking performance, the operator $Q$ with SVM is designed to early fault detection for the tube sensor fault $f_{w}$, while fault tolerant controller is used to the aluminum plate sensor fault $f_{a}$. The proposed control system is shown in Figure 9.

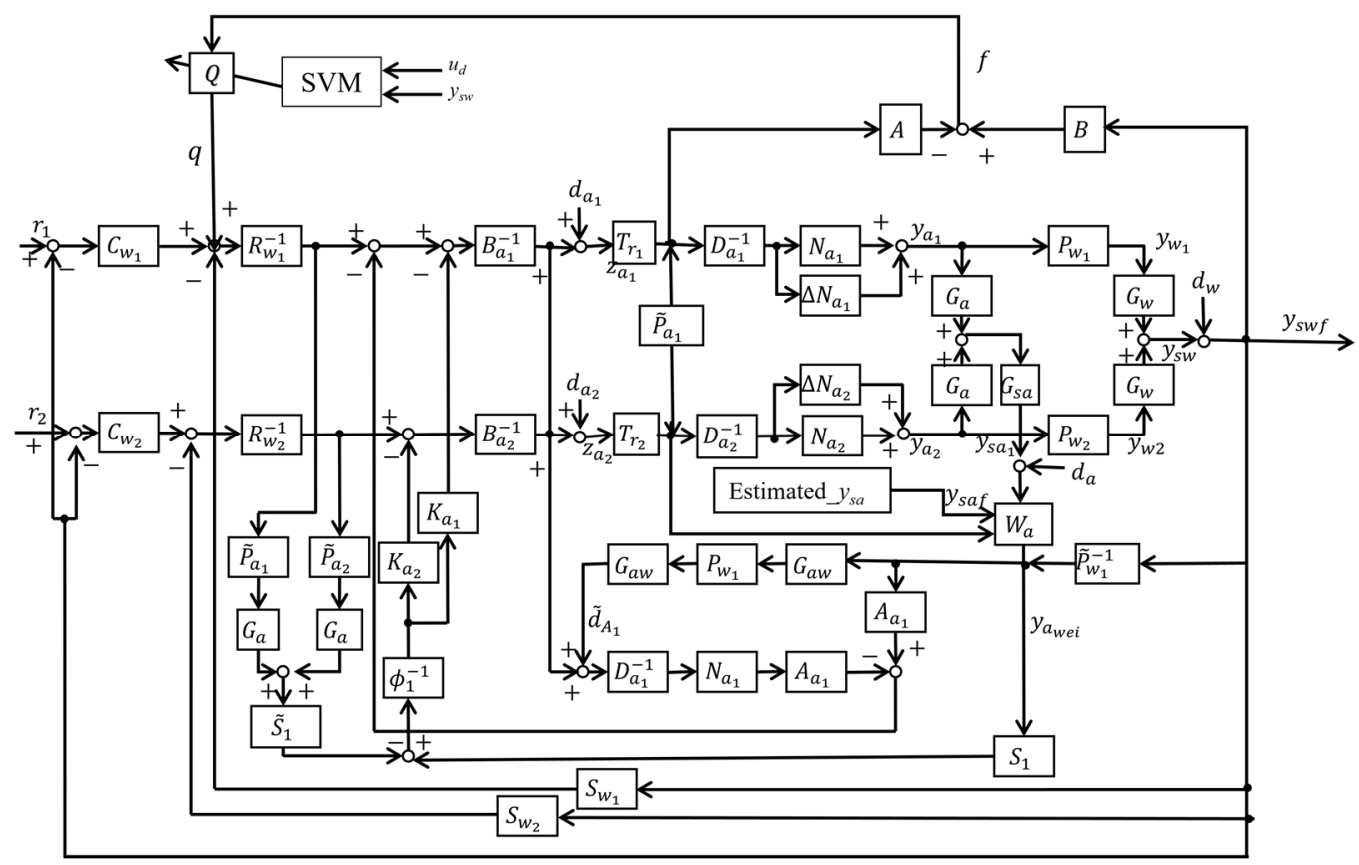

Figure 9. The proposed control system.

\section{Simulation and Experimental Tests}

Parameters of simulation and experiment are listed in Table 3.

Table 3. Parameter of simulation and experiment.

\begin{tabular}{cc}
\hline Parameters & Value [Units] \\
\hline Initial temperature & $25.0\left[{ }^{\circ} \mathrm{C}\right]$ \\
Reference input $r$ & $1.0\left[{ }^{\circ} \mathrm{C}\right]$ \\
Sampling time & $0.5[\mathrm{~s}]$ \\
Simulation time & $600[\mathrm{~s}]$ \\
Gain of $C_{K_{P}}$ & 0.3 \\
Gain of $C_{K_{I}}$ & 0.008 \\
Gain of $M_{K_{P}}$ & 0.45 \\
Gain of $M_{K_{I}}$ & 0.008 \\
Designed SVM parameter $\mathrm{C}$ & 1 \\
Designed SVM parameter $\gamma$ & 0.25 \\
\hline
\end{tabular}


Assume the case study is the added fault $4\left[{ }^{\circ} \mathrm{C}\right], T_{F}=300 \mathrm{~s}$, the fault signal $d_{a, w}$ is expressed as Equation (19):

$$
d_{a, w}=4\left[{ }^{\circ} \mathrm{C}\right] \times\left(1-\exp \left(-0.2\left(t-T_{F}\right)\right)\right)
$$

\subsection{Simulation Results}

The temperature sensor (tube) rises $4{ }^{\circ} \mathrm{C}$ in $300 \mathrm{~s}$ as a sensor fault signal shown in the first upper Figure. The second of Figure 10 presents the flag $=1$ when fault signal is detected at $304.5 \mathrm{~s}$ by using SVM. Compared to the conventional method without SVM algorithm which refers to Deng and Koyama [25], the fault is detected at $314.5 \mathrm{~s}$ shown as the dashed line in the third of Figure 10. The simulation results indicate that operator $Q$ with SVM classier is detected $10 \mathrm{~s}$ earlier by comparison on the conventional method without SVM classier. From the bottom of Figure 10, the temperature output has presented a better performance of FDI in the case of sensor fault. Therefore, it cannot only maintain safety operation, but also shorten the delay time in fault detection against the uncertainties and noise in the simulation case.
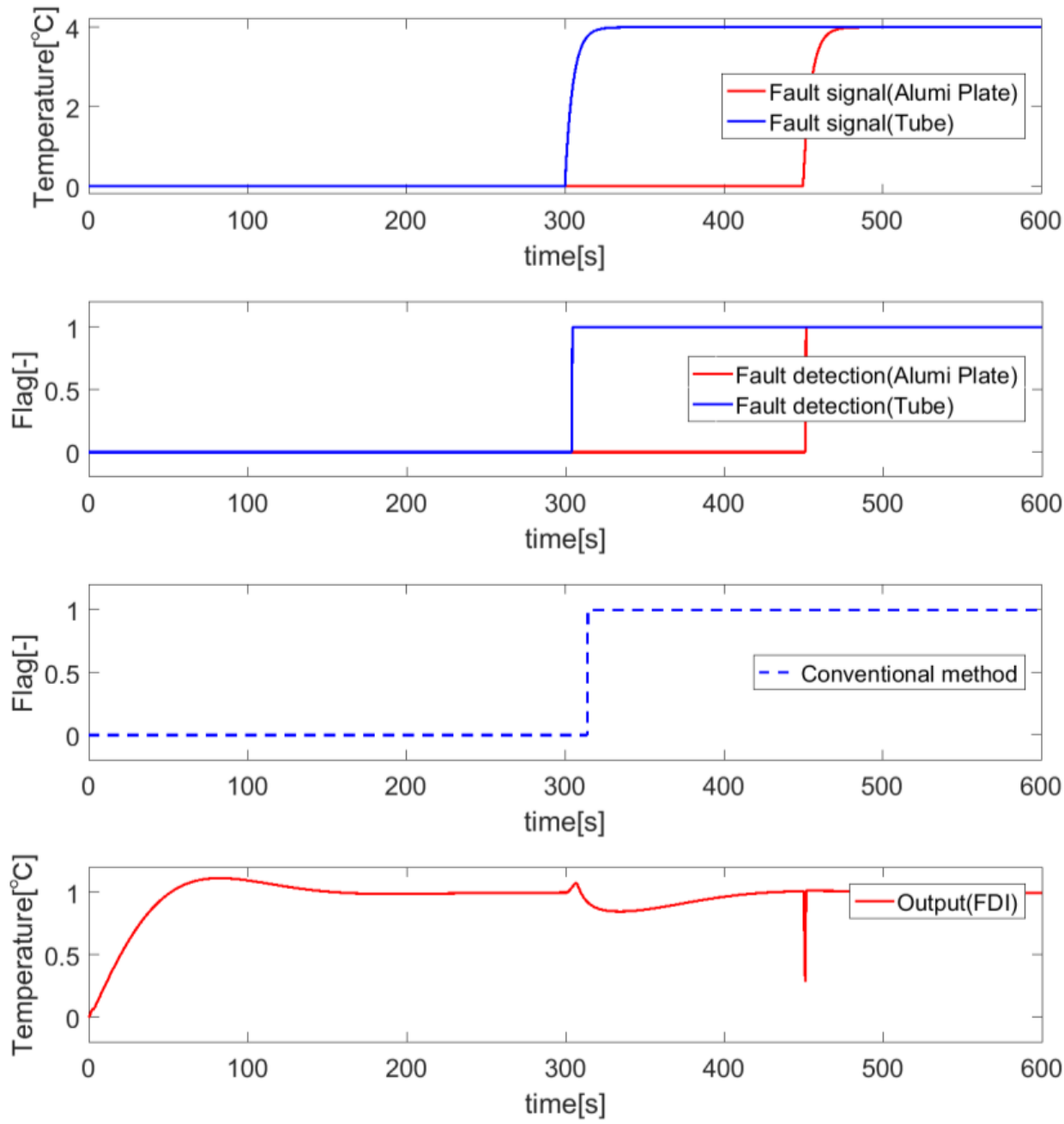

Figure 10. Simulation results.

From the viewpoint of control design, on the one hand, the conventional method in Deng and Koyama [25] consists of operator-based robust nonlinear control and fault tolerant control for one sensor fault $(\mathrm{i}=1,2)$. This work considers taking one step the previous research to improve the safety performance of FTC and extend to more sensor faults $(i=1,2,3)$. On the other hand, SVM as a binary classifier has been considered 
a flexible algorithm to perform accurate classification in dealing with noise-containing nonlinear process. The combination of FTC and SVM-based fault detection is to overcome the limitation of the conventional method. The comparative analysis of detection time has shown in simulation results with and without SVM-based fault detection [26].

\subsection{Experimental Tests}

Compared to the detection time in simulation, the experimental parameters for the setup were the same as the simulation parameters under one case study. The initial temperature was about $20^{\circ} \mathrm{C}$. The experimental tests are presented in Figures 11-13. In Figure 11, $d_{a}$ and $d_{w}$ indicate the fault signals of the aluminum plate sensor and the tube sensor in the upper picture, respectively, when the fault signals of $d_{a}$ and $d_{w}$ rise $4{ }^{\circ} \mathrm{C}$ in $300 \mathrm{~s}$ and $450 \mathrm{~s}$, respectively. In the middle picture, the multiple sensor faults can be detected within $5 \mathrm{~s}$. The output of FDI in the bottom of Figure 11 shows a good tracking performance under the faulty conditions. It is demonstrated that the proposed approach is flexible and effective to deal with the sensor faults. The temperature curves of the tube and aluminum plate are shown in Figure 12, while the input current is presented in Figure 13. The experiment results confirm that the tracking performance is improved by the proposed control system.

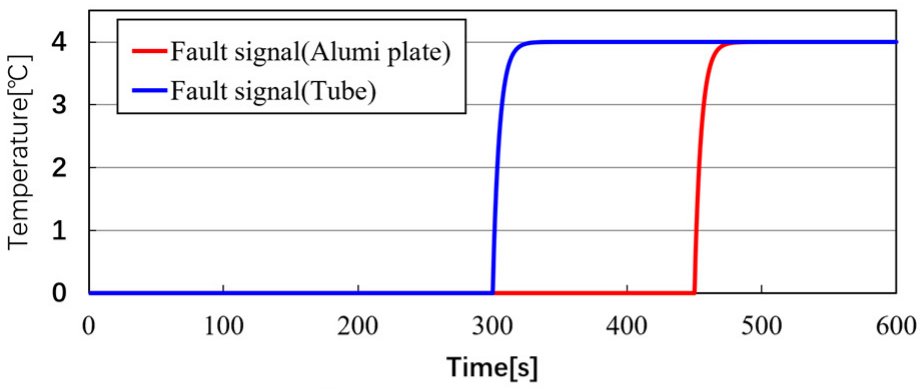

(1) Fault $d_{a}$ and fault $d_{w}$

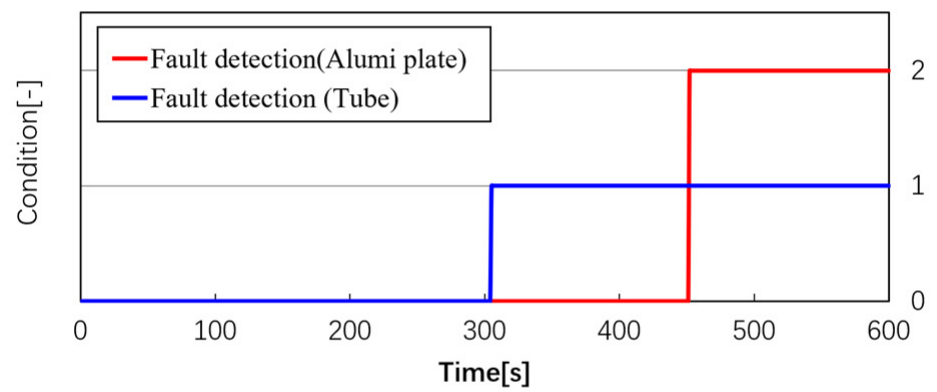

(2) Fault detection(tube sensor and aluminum plate sensor)

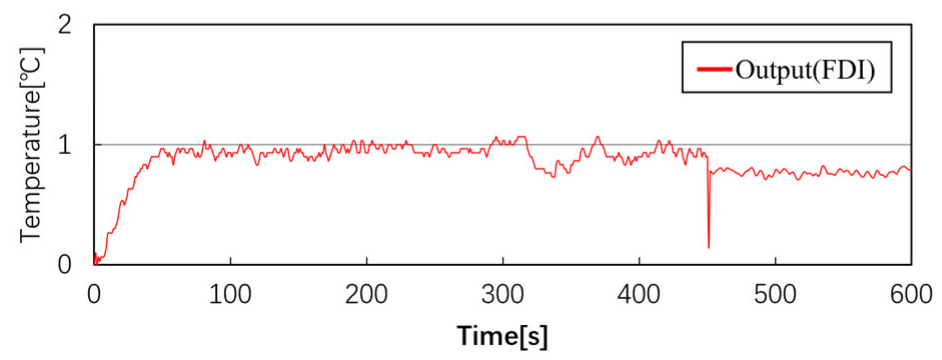

(3) Output of FDI

Figure 11. Experimental tests. 


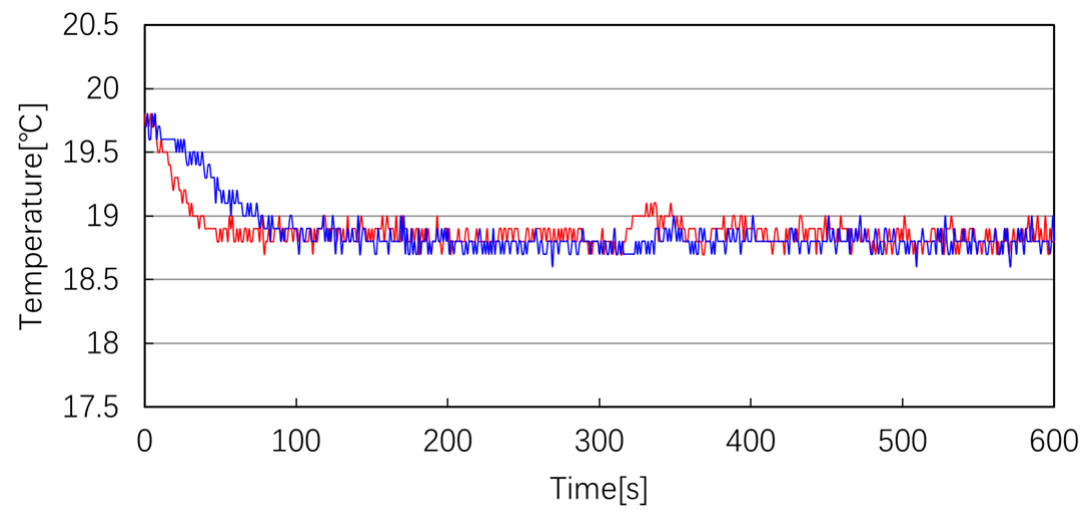

Figure 12. Temperature outputs of the tube and aluminum plate in the experiment.

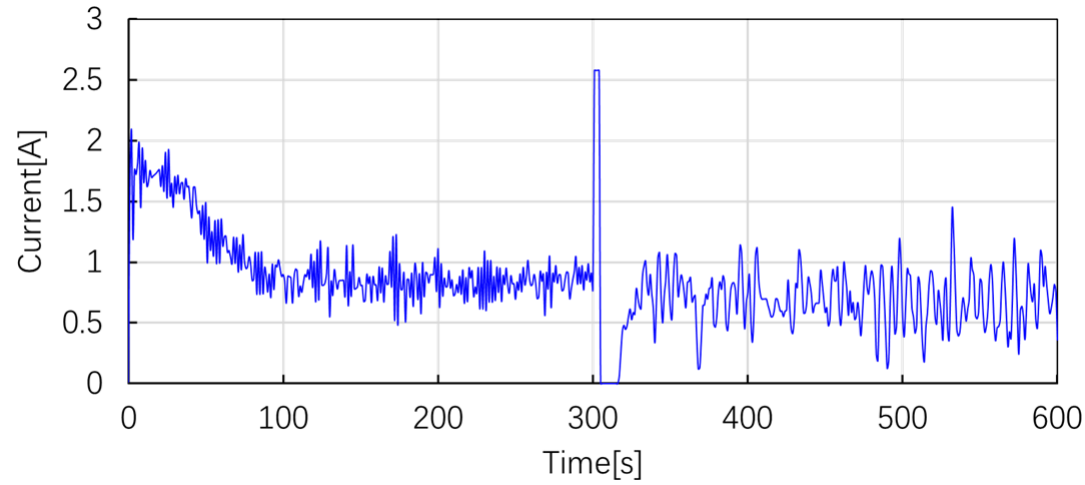

Figure 13. Input of the current in the experiment.

In this case study, given a desirable value $1.0{ }^{\circ} \mathrm{C}$, the output of the temperature is cooler than the initial temperature while the sensor faults were occurring. Considering the uncertainties and faults, both the robust stability and cooling performance were proven by the experiment test.

\section{Conclusions}

This paper presents a hybrid fault tolerant controller for a microreactor system subjected to sensor faults. For the parameter uncertainties and the effect of coupling, an operator-based nonlinear control design guarantees robust stability. Compared to the previous method without the SVM algorithm, operator Q with an SVM classier reduces the fault detection time when dealing with a noise-containing nonlinear process in the simulation results. The fault tolerant controller extends to cope with more sensor faults. A novel integrated fault tolerant controller with SVM-based early fault detection is proposed for the first time in order to maintain the safety operation in the event of sensor faults. The feasibility and effectiveness of the proposed FTC system are proven by the simulation and the experimental tests provide a good tracking performance in the case study. Future work will be on experimental tests for SVM classifier. In addition, the faults are often classified as actuator faults, sensor faults, and plant faults (or called component faults or parameter faults). The limitation of this work is the kind of sensor faults in the microreactor system, while the actuator and plant are assumed as the normal condition. Future work will focus on fault diagnosis, to determine the location, type, shape, and size of the faulty component in the event of the three types of faults. Moreover, we will consider the Lyapunov-based approach as well as these types of techniques to prove the stability and robustness of the model-based approach. 


\begin{abstract}
Author Contributions: Y.T. proposed the integrated fault tolerant controller with an SVM classifier for multiple sensor faults. X.L. wrote the manuscript. M.D. suggested technical support and gave overall guidance on the paper. All authors have read and agreed to the published version of the manuscript.
\end{abstract}

Funding: This research received no external funding.

Institutional Review Board Statement: Not applicable.

Informed Consent Statement: Not applicable.

Data Availability Statement: Data are contained within the article.

Conflicts of Interest: The authors declare no conflict of interest.

\title{
References
}

1. Deng, M.; Saijo, N.; Gomi, H.; Inoue, A. A robust real time method for estimating human multijoint arm viscoelasticity. Int. J. Innov. Comput. Inf. Control 2006, 2, 705-721.

2. Deng, M.; Inoue, A.; Zhu, Q. An integrated study procedure on real time estimation of time varying multijoint human arm viscoelasticity. Trans. Inst. Meas. Control 2011, 33, 919-941. [CrossRef]

3. Deng, M.; Iwai, Z.; Mizumoto, I. Robust Parallel Compensator Design for Output Feedback Stabilization of Plants with Structured Uncertainty. Syst. Control Lett. 1999, 36, 193-198. [CrossRef]

4. Bi, S.; Deng, M.; Xiao, Y. Robust Stability and Tracking for Operator-Based Nonlinear Uncertain Systems. IEEE Trans. Autom. Sci. Eng. 2015, 12, 1059-1066. [CrossRef]

5. Deng, M.; Wen, S.; Inoue, A. Operator-based robust nonlinear control for a Peltier actuated process, Measurement and Control. J. Inst. Meas. Control 2011, 44, 116-120. [CrossRef]

6. Deng, M.; Wang, A. Robust non-linear control design to an ionic polymer metal composite with hysteresis using operator-based approach. IET Control Theory Appl. 2012, 6, 2667-2675. [CrossRef]

7. Chen, G.; Han, Z. Robust right coprime factorization and robust stabilization of nonlinear feedback control systems. IEEE Trans. Auto. Control 1998, 43, 1505-1510. [CrossRef]

8. Deng, M.; Inoue, A.; Ishikawa, K. Operator-based nonlinear feedback control design using robust right coprime factorization. IEEE Trans. Auto. Control 2006, 51, 645-648. [CrossRef]

9. Gao, S.; Zhou, M.; Wang, Y.; Cheng, J.; Yachi, H.; Wang, J. Dendritic neuron model with effective learning algorithms for classification, approximation, and prediction. IEEE Trans. Neural Netw. Learn. Syst. 2018, 30, 601-614. [CrossRef] [PubMed]

10. Jin, G.; Deng, M. Operator-based robust nonlinear free vibration control of a flexible plate with unknown input nonlinearity. IEEE/CAA J. Autom. Sin. 2020, 7, 442-450. [CrossRef]

11. Wen, S.; Yu, J.; Guo, G.; Bi, S.; Wang, A.; Wang, D. Fractional order based robust right coprime factorization control for an inverted pendulum. In Proceedings of the 2016 International Conference on Advanced Mechatronic Systems, Melbourne, Australia, 30 November-3 December 2016; pp. 159-163.

12. Jiang, C.; Chu, K.; Ueno, S. Static Analysis of a 3-link Object for Posture Maintenance Control by Nonprehensile Robot without Compensating Friction. In Proceedings of the 2020 International Conference on Advanced Mechatronic Systems, Hanoi, Vietnam, 10-13 December 2020; pp. 270-273.

13. Bu, N.; Chen, W.; Jin, L.; Zhao, Y. Robust control for uncertain nonlinear feedback system using operator-based right coprime factorization. IEEE/CAA J. Autom. Sin. 2019, 6, 824-829. [CrossRef]

14. Deng, M.; Jiang, J.; Inoue, A.; Su, C. Operator-based robust control for nonlinear systems with Prandtl-Ishlinskii hysteresis. Int. J. Syst. Sci. 2011, 42, 643-652. [CrossRef]

15. Deng, M.; Inoue, A.; Goto, S. Operator based thermal control of an aluminium plate with a Peltier device. In Second International Conference on Innovative Computing, Informatio and Control (ICICIC 2007); IEEE: Piscataway, NJ, USA, 2008; pp. 3219-3229.

16. Wen, S.; Deng, M. Operator-based robust nonlinear control and fault detection for a Peltier actuated thermal process. Math. Comput. Model. 2013, 57, 16-29. [CrossRef]

17. Kawahata, S.; Deng, M. Operator-based Nonlinear Temperature Control Experiment for Microreactor Group Actuated by Peltier Devices. Int. J. Autom. Comput. 2016, 13, 401-408. [CrossRef]

18. Nguyen, N.P.; Nguyen, X.M.; Sung, K.H. Actuator Fault Detection and Fault-tolerant Control for Hexacopter. Sensors 2019, 19, 4721. [CrossRef] [PubMed]

19. Salahshoor, K.; Kordestani, M.; Khoshro, M.S. Fault detection and diagnosis of an industrial steam turbine using fusion of SVM (support vector machine) and ANFIS (adaptive neuro-fuzzy inference system) classifiers. Energy 2010, 35, 5472-5482. [CrossRef]

20. Furukawa, Y.; Deng, M. Fault detection of tank-system using ChangeFinder and SVM. In Proceedings of the 2020 International Conference on Advanced Mechatronic Systems, Hanoi, Vietnam, 10-13 December 2020; pp. 260-265.

21. De Figueiredo, R.; Chen, G. Nonlinear Feedback Control Systems-An Operator Theory Approach; Academic Press: San Diego, CA, USA, 1993.

22. Jiang, L.; Deng, M.; Inoue, A. Obstacle avoidance and motion control of a two wheeled mobile robot using svr technique. Int. J. Innov. Comput. Inf. Control 2009, 5, 253-262. 
23. Wen, S.; Deng, M.; Inoue, A. Operator-based robust non-linear control for gantry crane system with soft measurement of swing angle. Int. J. Model. Identif. Control 2012, 16, 86-96. [CrossRef]

24. Ogihara, Y.; Deng, M.; Noge, Y. Operator-based nonlinear fault detection and fault tolerance control for microreactor with generalized Gaussian function. In Proceedings of the 2019 1st International Conference on Industrial Artificial Intelligence (IAI), Shenyang, China, 23-27 July 2019; pp. 1-6.

25. Deng, M.; Koyama, A. Operator-based Robust Fault tolerant control for Uncertain Nonlinear Microreactors with Coupling Effects. In Proceedings of the 2019 American Control Conference (ACC), Philadelphia, PA, USA, 10-12 July 2019; pp. 3746-3751.

26. Deng, M.; Tanaka, Y.; Li, X. Operator-Based Robust Fault Tolerant Control with SVM-based Early Detection for Sensor Faults. In Proceedings of the 2021 International Conference on Advanced Mechatronic Systems, Tokyo, Japan, 9-12 December 2021; pp. 37-40. 